\title{
The use of the constrained prosthesis in the difficult primary knee arthroplasty
}

\author{
${ }^{\star 1,2}$ Vitalie Iacubitchii, ${ }^{1,2}$ Alexandru Betisor, ${ }^{1}$ Nicolae Erhan \\ ${ }^{1}$ Department of Orthopedics and Traumatology, Nicolae Testemitanu State University of Medicine and Pharmacy \\ ${ }^{2}$ Large Joints Replacement Department, Clinical Hospital of Traumatology and Orthopedics \\ Chisinau, the Republic of Moldova
}

Authors' ORCID iDs, academic degrees and contributions are available at the end of the article

${ }^{\star}$ Corresponding author - Vitalie Iacubitchii, e-mail: vitalie.iacubitchii@usmf.md

Manuscript received August 12, 2021; revised manuscript 04, 2021; published online November 26, 2021

\begin{abstract}
Background: The constrained knee prosthesis has the basic indication in revision arthroplasty, but the latest literature reveals that it takes place also in primary total knee arthroplasty in cases of knee osteoarthritis associated with major deformities.

Material and methods: Present study is based on the surgical treatment, using the constrained knee prosthesis in the primary total knee arthroplasty, during 2019-2021, of 28 patients with knee osteoarthritis associated with severe deformities in varus or valgus, in the Big Joint Replacement Department, Clinical Hospital of Traumatology and Orthopedics, Chisinau.

Results: In this study, the following criteria were evaluated: the type of deformity - valgus (10 cases) and varus ( 18 cases); the degree of deformation - for varus knees was on average $30^{\circ}$, and for valgus knee $-25^{\circ}$; bone attrition - 11 cases with bone defects where it was necessary to use augmentations; affected side - in 19 cases the right knee was affected and 9 cases the left one; the women/men ratio was $4 / 1$; the mean age of the patients was 67.5 years; average duration of the intervention - 140 minutes; in 5 cases a lateral para-patellar approach was performed, of which 2 cases with tibial tuberosity osteotomy; complications -1 case with intra-operative periprosthetic fracture and 2 cases with superficial infections of the operated joints.

Conclusions: Osteoarthritis of the knee progresses rapidly, leading to severe deformities, significant bone defects and joint instability, which are indications to use the constrained prosthesis in the primary total knee arthroplasty, long-term follow-up is necessary to obtain the last conclusion, but from this study the constrained knee prosthesis like primary implant for special indication had promising results.

Key words: knee osteoarthritis, difficult arthroplasty, constrained prosthesis.
\end{abstract}

Cite this article

Iacubitchii V, Betisor A, Erhan N. The use of the constrained prosthesis in the difficult primary knee arthroplasty. Mold Med J. 2021;64(5):11-15. https:// doi.org/10.52418/moldovan-med-j.64-5.21.02.

\section{Introduction}

Knee osteoarthritis (KOA) is a common progressive multifactorial joint disease and is characterized by chronic pain and functional disability [1]. KOA accounts for almost four-fifths of the burden of osteoarthritis worldwide and increases with obesity and age [2]. There were nearly 654 million individuals (40 years and older) with knee osteoarthritis in 2020 worldwide [3]. Up to now, KOA is incurable except knee arthroplasty which is considered an effective treatment at an advanced stage of the disease, however, which is responsible for substantial health costs [4].

Total knee arthroplasty (TKA) is a great success nowadays in modern orthopedics $[5,6]$ and is a procedure to restore proper function and give pain relief in patients with severe knee osteoarthritis [7].

The use of the constrained prosthesis in the primary TKA was unusual in all the world nearly 15 years ago [8], several years ago the interest in this method appeared in Moldova as well.

The constrained knee prosthesis (CKP) has the basic indication in revision arthroplasty, but the latest literature reveals that it takes place also in primary total knee arthroplasty in cases of severe knee osteoarthritis associated with major deformity with a significant bone defect, stiffness and instability [9]. CKP with its variety of available stems and augments can also help surgeons improve implant stability, optimum alignment, adequate balance and deformity correction [9-12]. Stability is essential for successful TKA [13-16]. By Sabatini et al. the rate of complication is decreased and a good survival rate and functional score results are shown by using the constrained condylar knee prosthesis in primary knee arthroplasty $[7,12,13]$.

But anyway, there are some possible disadvantage of CKP which include large bone removal, mechanical loosening due to load transfer to the respective bone ends through an intramedullar extension of the stems leading to early failure and a periprosthetic fracture $[9,14,17]$. Polyethylene insert wearing is another pitfall of CKP [14]. Revision of TKA following CKP is an extremely difficult procedure, as a need for stem removal increases significantly morbidity and operating time [9, 17]. Second generation condylar constrained knee (CCK) prosthesis 
reduced some complications to the patella (e.g., fractures, incorrect tracking and osteonecrosis) due to redesigned patellofemoral surfaces $[12,18]$.

The study aimed to evaluate the method of surgical treatment with constrained prosthesis used in the difficult primary TKA in the clinic.

\section{Material and methods}

The study is based on the surgical treatment, using the constrained knee prosthesis (CKP) in the primary total knee arthroplasty (TKA), between May 2019 and June 2021, of 28 patients with severe knee osteoarthritis (KOA) associated with major deformities, significant bone defect, stiffness and instability, in the Big Joint Replacement Department, Clinical Hospital of Traumatology and Orthopedics, Chisinau.

All patients were over 58 years old, with an average age of $67.5 \pm 9.95$ years $(58-77)$, there were 22 women and 6 men. Nineteen patients had right knee involvement, and 9 - left knee involvement. The mean body mass index (BMI) of the patients was $31.07 \pm 1.38$ (22.49-39.66). Detailed characteristics of the patients are illustrated in table 1 .

Table 1. Characteristics of patients

\begin{tabular}{|l|c|c|}
\hline \multicolumn{1}{|c|}{ Demographic parameters (N-28) } & Mean \pm SD & Range \\
\hline Age (years) & $67.5 \pm 9.95$ & $58-77$ \\
\hline Women/Men & $22 / 6$ & \\
\hline Right/left & $19 / 9$ & \\
\hline BMI $\left(\mathrm{kg} / \mathrm{m}^{2}\right)$ & $31.07 \pm 1.38$ & $22.49-39.66$ \\
\hline
\end{tabular}

$\mathrm{N}$ - total sample, BMI - body mass index, SD - standard deviation

The decision to use constrained knee prosthesis was taken pre-operatively in all 28 cases based on the severity of knee osteoarthritis - the major deformities, important bone loss and complex instability assessed clinically and radiographically (fig. 1).
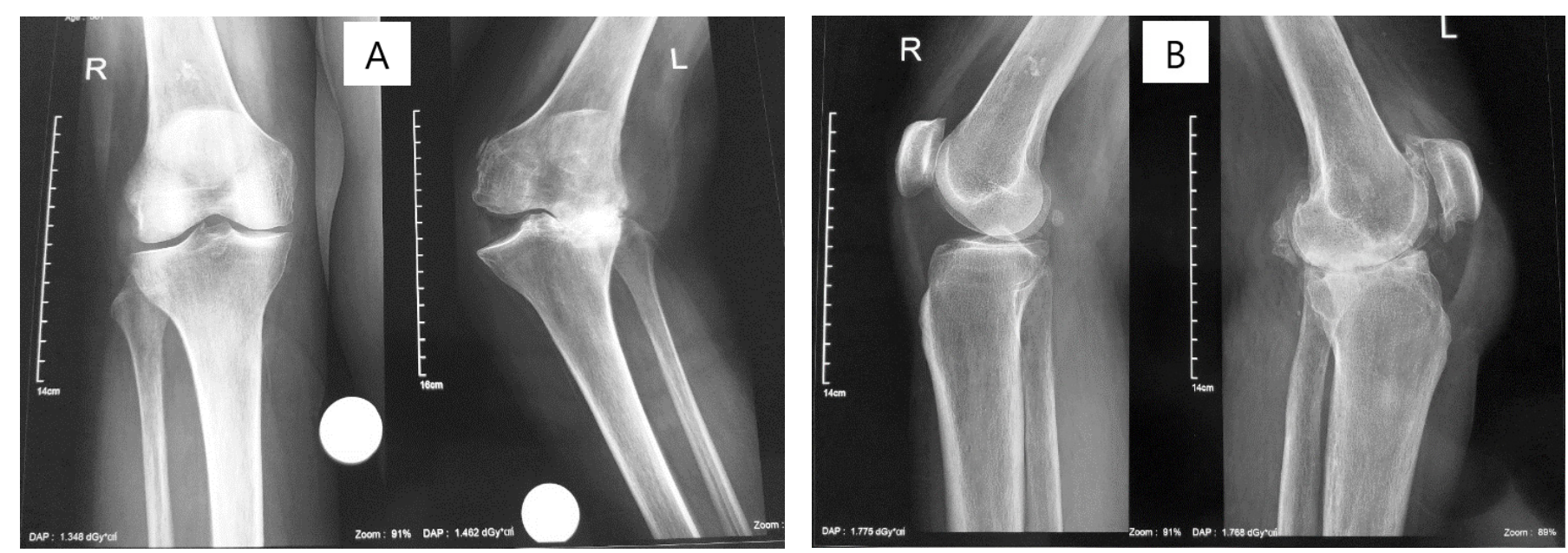

Fig. 1. Pre-operative radiographs: A - Anteroposterior (AP) view, B - Lateral view.
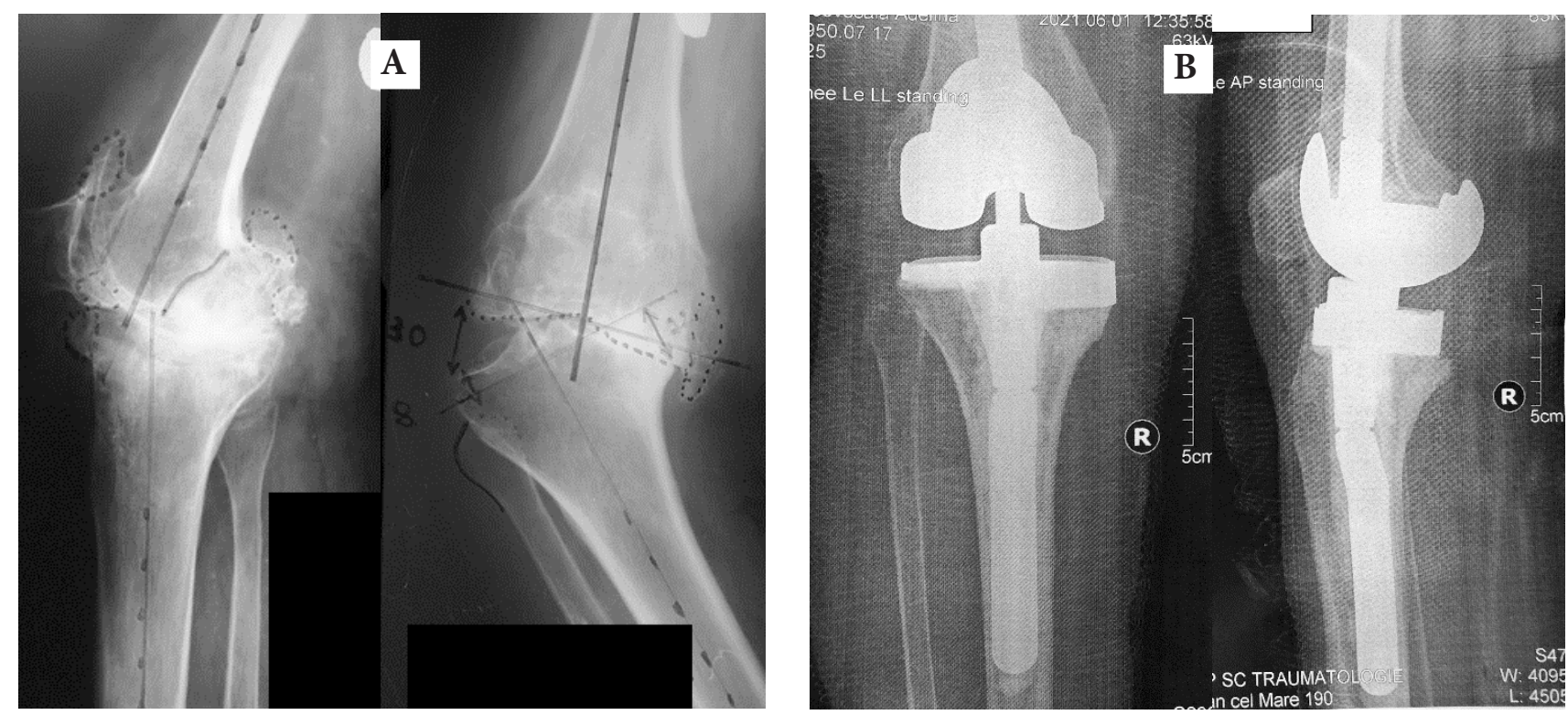

Fig. 2. CKP with augmentation on medial plateau implanted in a 70 -year-old woman because of $28^{0}$ varus deformity of the right knee. A - Pre-operative radiographs, B - Radiographs after surgery 
Pre-operative planning was made on all 28 knees. The constrained TKA was performed in knees with a varus over $20^{\circ}$ or valgus over $15^{\circ}$. Eighteen of 28 knees had a varus deformity (fig. 2) and 10 had a valgus deformity (fig. 3). The degree of deformation for varus knees was on average $30^{\circ}$ (ranging from $20^{\circ}$ to $40^{\circ}$ ), and for valgus knee $-25^{\circ}$ (ranging from $15^{0}$ to $35^{\circ}$ );

Another indication for the use of CKP is the advanced bone defect, based on Ahlbäck classification [19]:

1. Grade I: joint space narrowing (less than $3 \mathrm{~mm}$ ).

2. Grade II: joint space obliteration.

3. Grade III: minor bone attrition $(0-5 \mathrm{~mm})$.
4. Grade IV: moderate bone attrition (5-10 mm).

5. Grade V: severe bone attrition (more than $10 \mathrm{~mm}$ ).

Eleven cases were with severe bone loss and it was necessary to use augmentations, with thickness from 5 to 15 mm, respectively 9 on the medial (fig. 2 ), 1 on the lateral (fig. 3), and 1 on the entire surface of the tibial plateau. In one case, with moderate bone attrition, was used 1 screw on the medial compartment for better support of the tibial component (fig. 4).

The mean operative time was 140 min (85-195). A pneumatic tourniquet was used during the surgeries, when the allowed time had elapsed; the tourniquet was deflated for a short period, then inflated again.
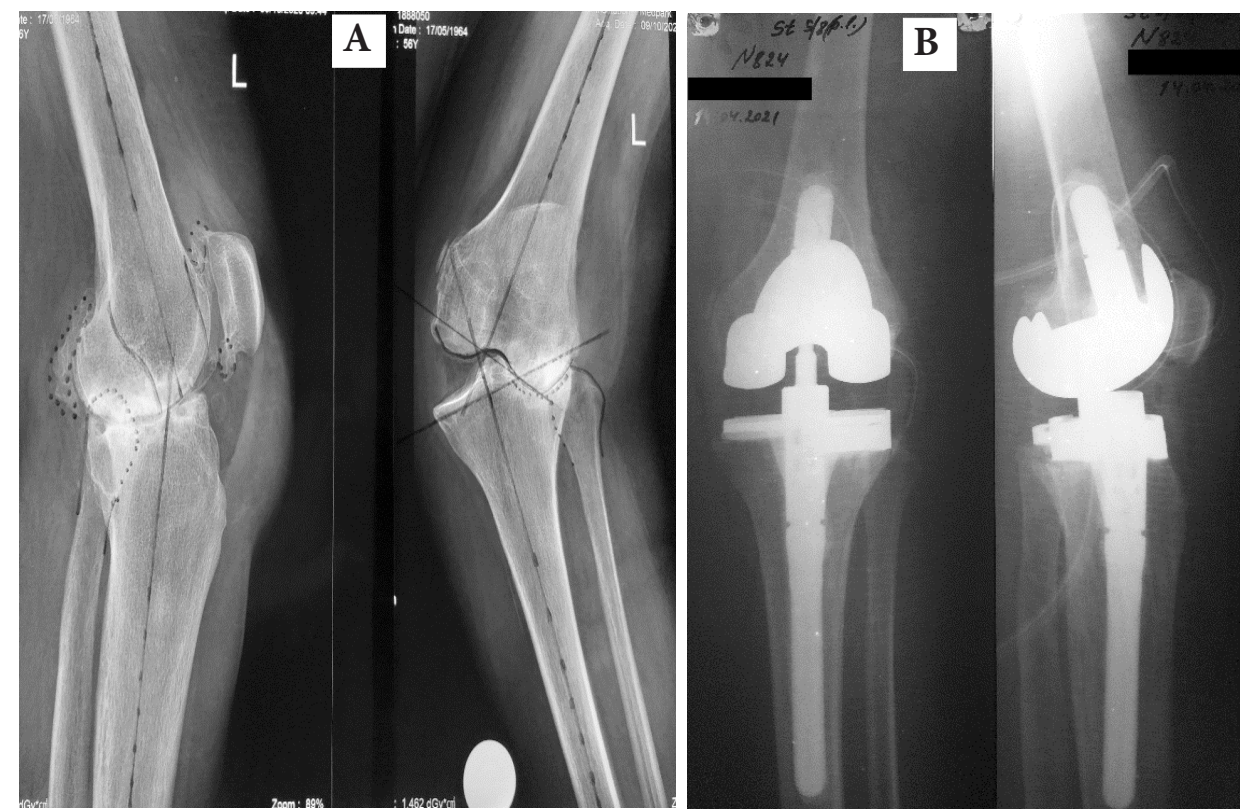

Fig. 3. CKP with augmentation $10 \mathrm{~mm}$ on lateral plateau implanted in a 56-year-old woman with a valgus deformity of $30^{\circ}$ of the left knee. A - Pre-operative radiographs, B - Radiographs after surgery
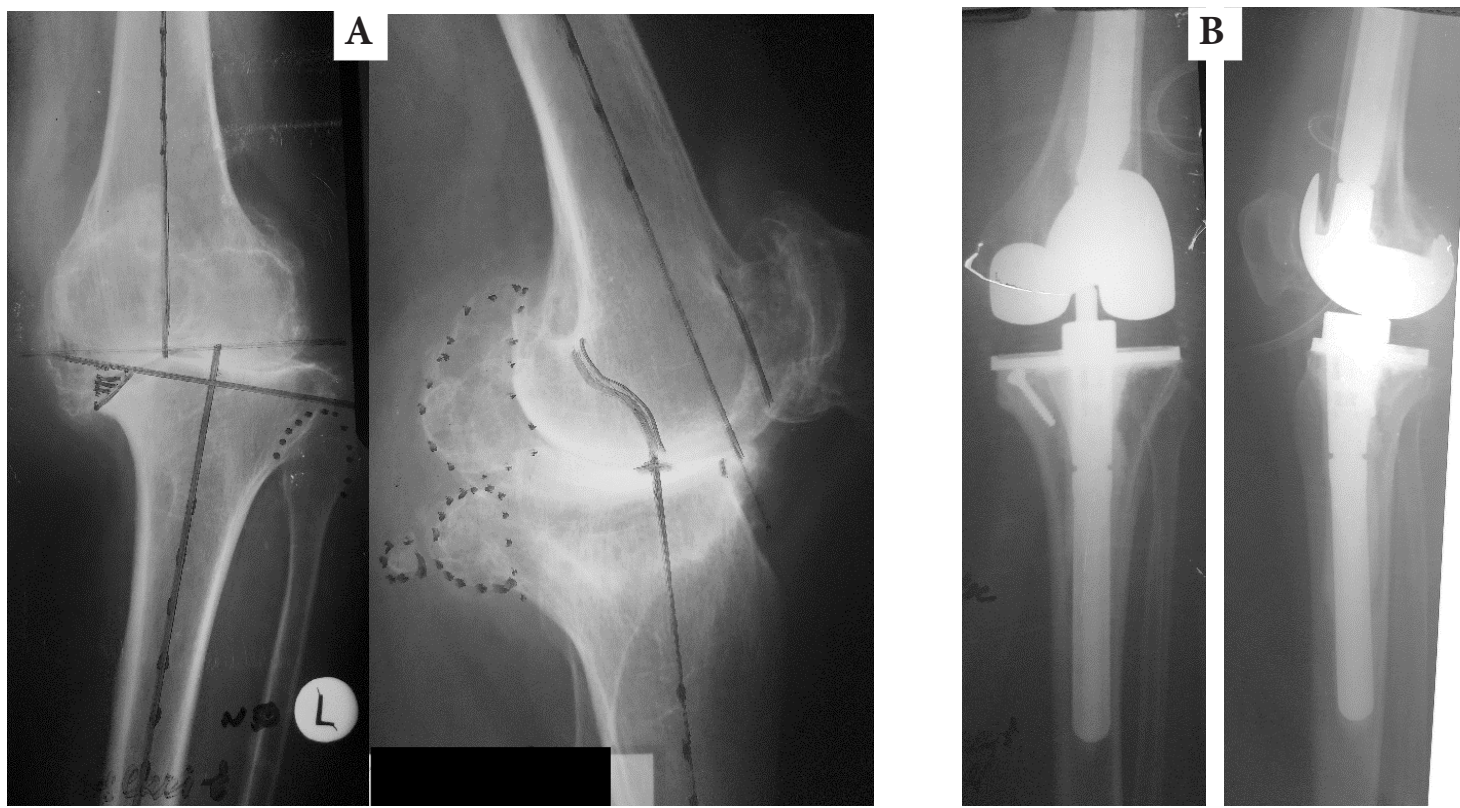

Fig. 4. CKP in a 76-year-old patient with a varus deformity of $15^{\circ}$ of the left knee. A - Pre-operative radiographs, B - Radiographs after surgery 

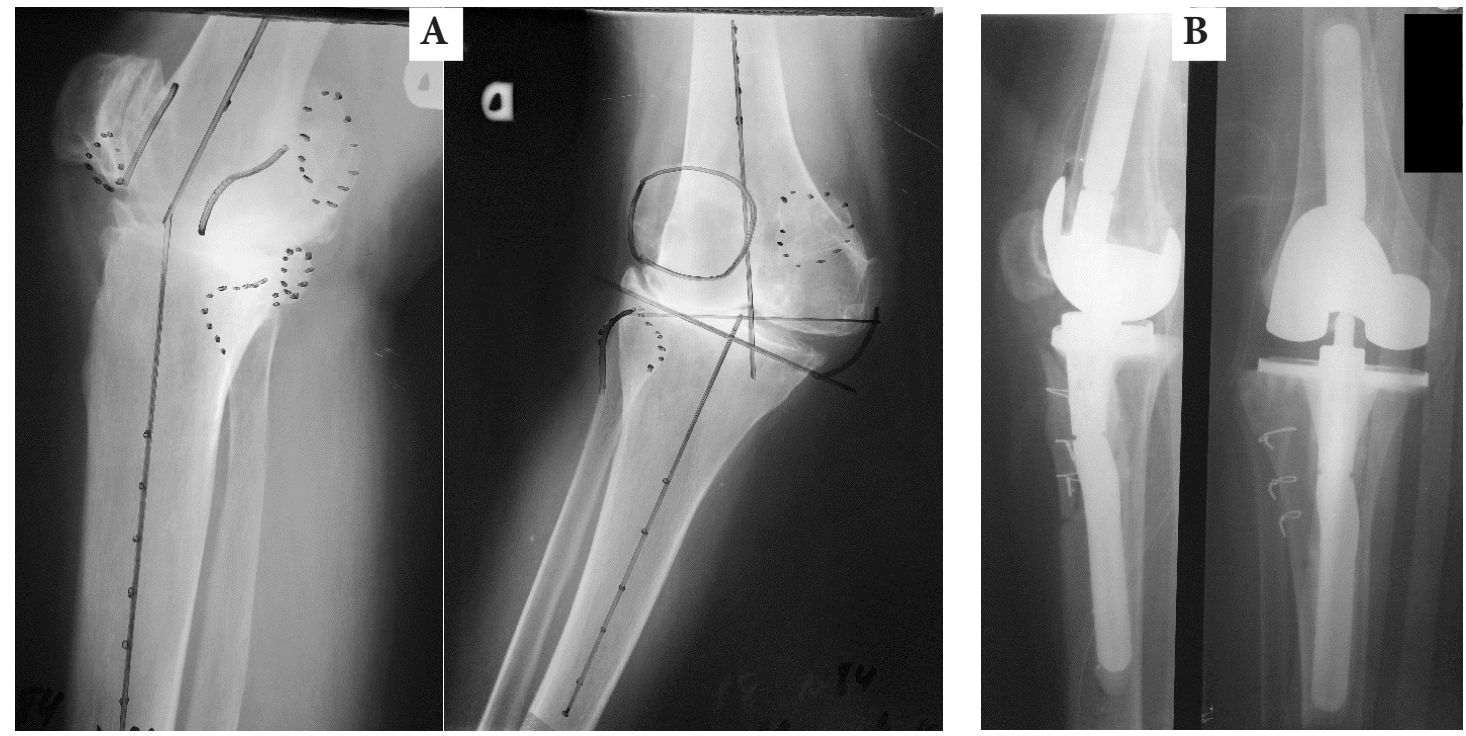

Fig. 5. CKP in a 67-year-old patient with a valgus deformity of $23^{\circ}$ of the right knee. A - Pre-operative radiographs, B - Radiographs after surgery

The following approaches were used: medial parapatellar - 21 cases; lateral as described by Keblish [20] - 5 cases; and mid-vast [21] - 2 cases (in moderate knee deformations). To achieve sufficient exposure in 2 cases were performed tibial tuberosity osteotomy (TTO) (fig. 5). The plasty of the articular capsule involving part of Hoffa's fat pad was performed in 3 cases with severe valgus deformity.

In one case, intra-operatively with periprosthetic lateral femoral condyle fracture, the result was achieved by osteosynthesis with one screw. In another case, intra-operatively has been determined on medial femoral condyle a subchondral cyst, size $2 \times 3 \times 2.5 \mathrm{~cm}$, which was supplanted with autologous bone grafting.

The intramedullary femoral and tibial guide was used routinely, stem extensions were always used and all components were cemented. Routine patella resurfacing was not performed in these cases; however, patella denervation with electrocautery was performed in all 28 knees.

\section{Results}

28 knees with constrained knee prosthesis were reviewed as the first implant. Ten patients were evaluated clinically and radiologically at 2-, 6-, 12-month after surgery, 14 patients at 2-, 6-month after surgery, and 4 patients at 2-month after surgery.

Knee Society Score (KSS) functional score was used to assess the patients [22]. The mean KSS improved from 25 points pre-operatively to 91 (74-100) points at the last follow-up. All patients recovered full extension during the follow-up.

Radiographs showed no radiolucent lines in all knees neither within the femur nor within the tibia. No component loosening or periprosthetic fracture was reported after the surgery. There were 2 cases with superficial infections of the operated joints, carried out by early irrigation and debridement (I\&D).
Five patients suffered from thigh pain, solved after 2-3 months of physiotherapy and rehabilitation. No revisions or reoperations were performed. 16 patients experienced numbness on the lateral side of the knee. There were no important neurovascular injuries in this experience.

\section{Discussion}

The need of a semi-constrained implant in primary TKA is rare due to ligament instability or significant bone defects; different recent works yet recommend to take into account the use of a CKP when it is particularly complex to gain adequate soft tissue balance. Insall et al. (1976) and Donaldson et al. (1988) had already described indications to CKP replacement among which are included severe axial deformities, collateral ligaments insufficiency and severe bone loss [12].

Negatives about the use of CKP include larger bone removal, polyethylene insert wearing, mechanical loosening due to extension of the stems which lead to early failure and a periprosthetic fracture $[9,14,17]$.

The most important deduction of this study was that the use of the constrained prosthesis in the primary total knee arthroplasty corrected the severe deformity with major bone defects, stiffness, and instability of the knee joint restoring excellent clinical outcome and recovered the needed function.

Using KSS after CKP, 11 cases were rated as excellent and 17 as good. Considering this report, it can be concluded that $\mathrm{CKP}$ as a primary implant is effective and justified option for the treatment of difficult KOA. However, it's not without some complications.

\section{Conclusions}

Osteoarthritis of the knee progresses rapidly, leading to severe deformities, significant bone defects and joint insta- 
bility, which are indications to use the constrained prosthesis in the primary total knee arthroplasty, long-term followup is necessary to obtain the last conclusion, but from the present study the constrained knee prosthesis like primary implant for special indication had promising results.

Rigorous selection of patients, pre-operation planning, compliance with surgical techniques according to the algorithm allows to obtain good functional results in the majority of cases.

Constrained prosthesis in the primary total knee arthroplasty allows the correction of deformity, gives stability, removing the pain syndrome, improving mobility in the joint, the relatively rapid resumption of function during the postoperative period and considerably improves the quality of life at the patients.

\section{References}

1. Hunter DJ, Bierma-Zeinstra S. Osteoarthritis. Lancet. 2019;393(10182): 1745-1759. doi: 10.1016/S0140-6736(19)30417-9.

2. Vos T, Allen C, Arora M, et al. Global, regional, and national incidence, prevalence, and years lived with disability for 310 diseases and injuries, 1990-2015: a systematic analysis for the global burden of disease study 2015. Lancet. 2016;388(10053):1545-1602. doi: 10.1016/S01406736(16)31678-6.

3. Aiyong C, Huizi L, Dawei W, Junlong Z, Yufeng C, Huading L. Global, regional prevalence, incidence and risk factors of knee osteoarthritis in population-based studies. EClinicalMedicine. 2020;29-30:100587. doi: 10.1016/j.eclinm.2020.100587.

4. Bannuru RR, Osani MC, Vaysbrot EE, et al. OARSI guidelines for the non-surgical management of knee, hip, and polyarticular osteoarthritis. Osteoarthritis Cartilage. 2019;27(11):1578-1589. doi: 10.1016/j. joca.2019.06.011.

5. Darciuc M. Tratamentul chirurgical al fracturilor patelei [Knee arthroplasty problems]. [Bull Acad Sci Mold Med Sci]. 2009;21(2):163-166. Romanian. doi: 10.52692/1857-0011.

6. Stratan L, Sirbu P. Classification and the history of total knee arthroplasty. Rev Ortoped Traumatol (Bucuresti). 2004;14(3-4):191-200.

7. Sabatini L, Risitano S, Atzori F, Aprato A, Massè A. Indications and results of condylar constrained implant in primary total knee replacement. Ann Orthop Musculoskelet Disord. 2017;1(1):1005. doi: 10.21037/ atm.2017.03.29.

8. Lachiewicz PF, Soileau ES. Ten-year survival and clinical results of constrained components in primary total knee arthroplasty. J Arthroplasty. 2006;21(6):803-8. doi: 10.1016/j.arth.2005.09.008.
9. Rai S, Liu X, Feng X, et al. Primary total knee arthroplasty using constrained condylar knee design for severe deformity and stiffness of knee secondary to post-traumatic arthritis. J Orthop Surg Res. 2018;13:67. doi: 10.1186/s13018-018-0761-x.

10. Bala A, Penrose CT, Seyler TM, Mather RC 3rd, Wellman SS, Bolognesi MP. Outcomes after total knee arthroplasty for post-traumatic arthritis. Knee. 2015;22(6):630-9. doi: 10.1016/j.knee.2015.10.004

11. Mozella Ade P, Olivero RR, Alexandre H, Cobra AB. Use of a trabecular metal cone made of tantalum, to treat bone defects during revision knee arthroplasty. Rev Bras Ortop. 2014;49(3):245-51. doi: 10.1016/j. rboe.2014.03.009.

12. Sabatini L, Risitano S, Rissolio L, Bonani A, Atzori F, Massè A. Condylar constrained system in primary total knee replacement: our experience and literature review. Ann Transl Med. 2017;5(6):135. doi: 10.21037/ atm.2017.03.29.

13. Sanguineti F, Mangano T, Formica M, Franchin F. Total knee arthroplasty with rotating-hinge Endo-Model prosthesis: clinical results in complex primary and revision surgery. Arch Orthop Trauma Surg. 2014;134(11):1601-7. doi: 10.1007/s00402-014-2061-1.

14. Cholewinski P, Putman S, Vasseur L, Migaud H, Duhamel A, Behal H, Pasquier G. Long-term outcomes of primary constrained condylar knee arthroplasty. Orthop Traumatol Surg Res. 2015;101(4):449-54. https:// doi.org/10.1016/j.otsr.2015.01.020.

15. Fehring TK, Odum S, Griffin WL, Mason JB, Nadaud M. Early failures in total knee arthroplasty. Clin Orthop Relat Res. 2001;392:315-8. doi: 10.1097/00003086-200111000-00041.

16. Clarke HD, Scuderi GR. Flexion instability in primary total knee replacement. J Knee Surg. 2003;16(2):123-8.

17. Anderson JA, Baldini A, MacDonald JH, Pellicci PM, Sculco TP. Primary constrained condylar knee arthroplasty without stem extensions for the valgus knee. Clin Orthop Relat Res. 2006;442:199-203. doi: 10.1097/01. blo.0000185675.99696.29.

18. Lachiewicz PF, Soileau ES. Results of a second-generation constrained condylar prosthesis in primary total knee arthroplasty. J Arthroplasty. 2011;26(8):1228-31. doi: 10.1016/j.arth.2011.05.010.

19. Ahlback S. Osteoarthrosis of the knee. A radiographic investigation Acta Radiol Diagn (Stockh). 1968;Suppl 227:7-72.

20. Keblish PA. The lateral approach to the valgus knee. Surgical technique and analysis of 53 cases with over two-year follow-up evaluation. Clin Orthop Relat Res. 1991;(271):52-62.

21. Engh GA, Holt BT, Parks NL. A midvastus muscle-splitting approach for total knee arthroplasty. J Arthroplasty. 1997;12(3):322-31. doi: 10.1016/ s0883-5403(97)90030-9.

22. Insall JN, Dorr LD, Scott RD, et al. Rationale of the Knee Society clinical rating system. Clin Orthop Relat Res. 1989;(248):13-4.

\section{Authors' ORCID iDs and academic degrees}

Vitalie Iacubitchii, MD, Assistant Professor - https://orcid.org/0000-0001-9465-7019

Alexandru Betisor, MD, PhD Associate Professor - https://orcid.org/0000-0003-2990-230X

Nicolae Erhan, MD, PhD, Associate Professor - https://orcid.org/0000-0003-3430-4531

\section{Authors' contributions}

VI designed the trial and interpreted the data. $\mathrm{AB}$ and NE revised the manuscript critically. All the authors approved the final version of the manuscript.

\section{Funding}

This study was supported by Nicolae Testemitanu State University of Medicine and Pharmacy. The trial was the authors' initiative. The authors are independent and take responsibility for the integrity of the data and accuracy of the data analysis.

\section{Ethics approval and consent to participate}

The research project was approved by the Research Ethics Committee of Nicolae Testemitanu State University of Medicine and Pharmacy (protocol No 02, of 23.10.2017).

\section{Conflict of Interests}

No competing interests were disclosed. 